Volumen 24, No 1, Páginas 41-48

IDESIA (Chile) Enero - Abril 2006

\title{
BIOLOGÍA Y ENEMIGOS NATURALES DE PEREGRINUS MAIDIS (ASHMEAD) (HEMIPTERA: DELPHACIDAE) EN EL VALLE DE AZAPA
}

\section{BIOLOGY AND NATURAL ENEMIES OF PEREGRINUS MAIDIS (ASHMEAD) (HEMIPTERA: DELPHACIDAE) IN THE AZAPA VALLEY}

\author{
Tommy C. Rioja ${ }^{1}$; Héctor E. Vargas ${ }^{1}$; Dante E. Bobadilla ${ }^{1}$
}

\begin{abstract}
RESUMEN
El delfácido del maíz, Peregrinus maidis, se encuentra presente en Chile en los valles costeros de Lluta y Azapa. Se realizó un estudio para caracterizar su ciclo de vida y conocer sus enemigos naturales en el agroecosistema local. Las observaciones fueron efectuadas en cultivos de maíz amiláceo, Zea mays L. var. amylacea Sturtevant, cv. Lluteño, ubicados en el campo experimental de la Facultad de Agronomía de la Universidad de Tarapacá, km. 12 del valle de Azapa. La hembra inserta los huevos en la nervadura central de las hojas y, en general, en los tejidos tiernos de la planta. El insecto presenta cinco estadios ninfales durante su desarrollo ontogénico. El primer instar con una duración de 10,05 $( \pm 1,28)$ días; el segundo instar 8,94 $( \pm 2,02)$ días; el tercer instar 9,94 $( \pm 1,03)$ días; el cuarto instar 10,35 $( \pm 1,73)$ días; el quinto instar $11,06( \pm 2,30)$ días; y el adulto $62,1( \pm 14,13)$ días de vida. Se pudo comprobar que los adultos presentan formas macrópteras y braquípteras. P. maidis se comporta como un monófago estricto, colectándose, eventualmente, sobre Setaria verticillata y Pitraea cuneato-ovata. Los enemigos naturales más frecuentes son Anagrus sp. (Mymaridae), que es parasitoide de huevos; y varios predadores generalistas, incluidas arañas (Araneae), Nabis sp. (Nabidae) y larvas de neurópteros (Chrysopidae y Hemerobiidae).
\end{abstract}

Palabras clave: Zea mays L., ambientes áridos, Peregrinus maidis, ciclo ontogénico, Anagrus sp.

\section{ABSTRACT}

The distribution of the corn delphacid, Peregrinus maidis, appears to be restricted in Chile to the coastal northernmost valleys, namely Lluta and Azapa. In order to know its life cycle and natural enemies under existing conditions in the local agroecosystem, a study was carried out during two phenological cycles of soft corn, Zea mays L. var. amylacea Sturtevant cv. Lluteño. The experimental crops were grown in the Campus of the Facultad de Agronomía, University of Tarapacá, km. 12 of Azapa valley. Planthopper female inserts its eggs in the young tissues around the central rib of the leaf. This delphacid undergoes five nymphal stadia during its ontogenetic development. The average duration of the nymphal instars was $10.05 \pm 1.28 ; 8.94 \pm 2.02 ; 9.94 \pm$ 1.03; $10.35 \pm 1.73$; and $11.06 \pm 2.30$ days for the first through fifth instars, respectively. The adult life span was $62.1 \pm 14.13$ days. It was proved that imagines exhibit wing dimorphism, being either macropterous or brachypterous morphotypes. In the corn biocoenosis of Azapa valley P. maidis is a strict monophagous species though adults were eventually collected on Setaria verticillata and Pitraea cuneato-ovata, two common weeds affecting corn crops here. The mostly observed natural enemies were Anagrus $s p$ (Mymaridae), an oophagous parasitoid, and many other general predators such as spiders (Araneae), Nabis sp. (Nabidae), Chrysoperla sp. (Chrysopidae), and brown lacewings (Hemerobiidae).

Key words: Zea mays L., arid environments, Peregrinus maidis, ontogenetic cycle, Anagrus $s p$.

\section{INTRODUCCIÓN}

El delfácido o "salta hojas" del maíz, Peregrinus maidis (Ashmead) (Hemiptera: Auchenorrhyncha: Delphacidae), es considerado entre las especies más nocivas al cultivo del maíz en los trópicos (King \& Saunders, 1984; Schmutterer, 1990; Alata, 1973; Cisneros, 1995); ya que, al alimentarse del floema, provoca daños directos e indirectos, causando amarillamiento (hopperburn) y enanismo arbustivo. P. maidis transmite las siguientes enfermedades virales: "Maize Stripe vi-

1 Laboratorio de Entomología, Facultad de Agronomía, Universidad de Tarapacá, Campus Azapa, Arica - Chile.

Fecha de Recepción: 04 Enero de 2006

Fecha de Aceptación: 03 Marzo de 2006 
rus", "Maize Mosaic virus", "Iranian maize Mosaic", "Maize raya gruesa rhabdovirus", "Maize line ", "Maize gooseneck stripe " y "Maize Sterile Stunt" (Cuezzo \& Virla, 2001). Es el único vector conocido del tenuivirus de la hoja rayada del maíz y del rhabdovirus del mosaico del maíz (Tsai \& Perrier, 1993).

Wilson \& Claridge (1991) señalan que P. maidis se encuentra en todas las áreas tropicales alrededor del mundo, alimentándose del sorgo o maíz. Asimismo, también se ha encontrado en caña de azúcar. Es una plaga conocida del sorgo, y no se conoce que se alimente de la planta del arroz, pero ocasionalmente es colectado en los alrededores de los cultivos de arroz.

Algunos autores, hoy en día, señalan a P. maidis dentro del ya antiguo Orden Homoptera (Borror et al. 1976; Marín \& Sarmiento, 1981; Fernández-Badillo \& Clavijo, 1990; Tsai \& Perrier, 1993; Remes Lenicov \& Virla, 1999). Actualmente, según Fletcher \& Larivière (2001); Cuezo \& Virla (2001); Carver et al. (1991), la ubicación taxonómica correcta para referirse a la familia Delphacidae, y por consiguiente al delfácido del maíz, es el Orden Hemiptera, Suborden Auchenorrhyncha. P. maidis se encuentra presente en los valles costeros de Lluta y Azapa (Aguilera, 1972). Por esta razón, se hace necesario conocer la biología y los enemigos naturales de este delfácido. Esta información ayudará en la implementación de un futuro manejo integrado de plagas, en el cultivo del maíz evitando, así, pérdidas económicas y la incorporación de productos tóxicos en el agroecosistema local.

\section{MATERIALES Y MÉTODOS}

Las observaciones de campo se realizaron entre agosto de 2002 y octubre de 2003, en dos cultivos sucesivos sembrados en el mismo terreno (con una superficie de $52 \mathrm{~m}^{2}$, la cual incluyó 4 camas de $1 \mathrm{~m}$. de ancho por $10 \mathrm{~m}$. de largo, con un total de 272 plantas en cada cultivo), perteneciente al Campo Experimental de la Facultad de Agronomía de la Universidad de Tarapacá, km 12 del valle de Azapa (18 $35^{\prime}$ ' Latitud Sur y 60³0' Longitud Oeste). No se aplicó pesticidas, excepto Bacillus thuringiensis var. kurstaki (Dipel®) para controlar Spodoptera frugiperda (J.E. Smith), minimizando de este modo las perturbaciones en la biocenosis del cultivo. Las mediciones sobre la duración del ciclo ontogénico fueron efectuadas sobre individuos aislados en 30 microjaulas (aros de tubo de PVC cada uno con un radio $=2 \mathrm{~cm}$, altura $=2 \mathrm{~cm}$, cubierta por muselina), ubicadas en el envés de las hojas y distribuidas en distintas plantas debidamente rotuladas. Los registros de temperatura y humedad relativa se obtuvieron de la Estación Climatológica Azapa, km 12, perteneciente a la Dirección General de Aguas, ubicada en la parcela $N^{\circ} 27$ de la Universidad de Tarapacá. En el Cuadro 1 se muestran las medias climáticas calculadas para cada etapa de desarrollo del insecto. A cada microjaula se transfirió un insecto recién eclosionado del huevo. Para tal efecto se colectaron hojas de maíz en las primeras horas de la mañana, en las cuales la oviposición se había producido ese mismo día. Posteriormente, estas hojas fueron llevadas a laboratorio para mantenerlas a una alta humedad relativa, y, así, evitar la deshidratación de las mismas, y asegurar de este modo la viabilidad de los huevos. Finalmente, al eclosionar los huevos, las ninfas recién emergidas fueron transportadas a sus respectivas microjaulas instaladas en plantas distribuidas al azar. Posteriormente, se determinó el tiempo exacto de la respectiva ecdisis, mediante la presencia del exuvio que deja el insecto al pasar de un estadio a otro.

En relación a los enemigos naturales, se colectó material vegetal periódicamente; una planta por camellón cada 15 días, depositándola posteriormente, al interior de una bolsa plástica de color negro; cada planta contenida se roció con un insecticida casero (Baygonâ, contiene 0,04\% de cyfluthrin; 0,5\% de diclorvos; Ingredientes inertes; Solvente; Propelente y c.p.s. 100\%), para facilitar su posterior revisión. El material colectado a través de este método fue conservado en alcohol al 70\%.

Además, se realizaron observaciones en los alrededores del cultivo de maíz, en la totalidad del campo experimental de la Facultad de Agronomía, valle de Azapa, como también en el valle de Lluta, para detectar posibles plantas hospedantes de $P$. maidis, incluyendo malezas gramíneas en general.

\section{RESULTADOS Y DISCUSIÓN}

Desarrollo Embrionario: La eclosión de los huevos en condiciones de laboratorio, con tempe- 
Cuadro 1

Registros Climáticos, Período de Observación (mayo-septiembre, 2003)

\begin{tabular}{|l|c|c|c|c|c|}
\hline Período & Etapa de Desarrollo & $\mathbf{T}^{\mathbf{o}}$ Mínima $\left({ }^{\mathbf{o}} \mathbf{C}\right)$ & $\mathbf{T}^{\mathbf{o}}$ Máxima $\left({ }^{\mathbf{o}} \mathbf{C}\right)$ & $\mathbf{T}^{\mathbf{o}}$ Media $\left({ }^{\mathbf{o}} \mathbf{C}\right)$ & H. R. $(\%)$ \\
\hline $02 / 05 / 03-24 / 06 / 03$ & Desarrollo Embrionario & 11,2 & 21,3 & 16,3 & 60 \\
$12 / 05 / 03-25 / 05 / 03$ & Ninfa I & 14,1 & 22,9 & 18,5 & 74,6 \\
$19 / 05 / 03-07 / 06 / 03$ & Ninfa II & 11,2 & 21,2 & 16,2 & 77,3 \\
$27 / 05 / 03-18 / 06 / 03$ & Ninfa III & 9,6 & 20,4 & 15 & 74 \\
$07 / 06 / 03-24 / 06 / 03$ & Ninfa IV & 9,6 & 20,5 & 15,1 & 72,6 \\
$16 / 06 / 03-08 / 07 / 03$ & Ninfa V & 9,6 & 19,8 & 14,7 & 74 \\
$26 / 06 / 03-24 / 09 / 03$ & Adulto & 11,2 & 19,8 & 15,5 & 74 \\
\hline
\end{tabular}

Fuente: Estación Climatológica Azapa, Km 12.

raturas medias que oscilaron entre los $11,2^{\circ} \mathrm{C}$ $21,3^{\circ} \mathrm{C}$, y con una alta humedad relativa cercana al $100 \%$, se produjo a los $22,68 \pm 1,83$ días.

Ninfas: Peregrinus maidis presenta 5 estadios ninfales (ver Cuadro 2). El primer instar se desarrolló en 10,05 $\pm 1,28 \mathrm{ds}$; el segundo instar 8,94 $\pm 2,02 \mathrm{ds}$; el tercer instar 9,88 $\pm 1,11 \mathrm{ds}$; el cuarto instar $10,35 \pm 1,73 \mathrm{ds}$ y el quinto instar $11,06 \pm 2,30$ ds. Además, sólo hubo mortalidad en los dos primeros instares; en el primer estadio ninfal se registró una mortalidad del $30 \%$, mientras que el segundo estadio ninfal se observó una mortalidad del 23,53\%. La mortalidad observada sugiere que los estadios ninfales son sensibles a la deshidratación y, por lo tanto, son afectados por bajos índices de humedad relativa, ya que, en ensayos de laboratorio a H.R. cercana al $100 \%$, la mortalidad fue casi nula; lo que también guarda relación con su comportamiento natural. Se debe mencionar además que, inicialmente, las microjaulas fueron instaladas en la cara adaxial de las hojas de maíz, produciéndose una mortalidad del $100 \%$ de los individuos, por lo que se optó en repetir el ensayo; así, nuevamente fueron instaladas las microjaulas, pero esta vez en la cara abaxial de las hojas de maíz, para atenuar, en cierto modo, los efectos de la alta radiación solar y de los vientos presentes en la zona, que causan una alta deshidratación, demostrando fehacientemente que los primeros estadios ninfales de $P$. maidis son sensibles a dichas variables climáticas.

Adulto: La longevidad del adulto promedió $62,1 \pm 14,13 \mathrm{ds}$, en condiciones de cautiverio en el valle de Azapa; estos resultados se refieren a la longevidad fisiológica de los individuos, ya que la longevidad ecológica es mucho menor, por tener variantes climáticas, biológicas y especialmente de enemigos naturales del delfácido en el agroecosistema local.

Cópula: La cópula se lleva a cabo en horas de la mañana y en la tarde, cuando las condiciones climáticas son más favorables, demorando alrededor de 40-60 minutos en completarse. El morfotipo alar no influye en el comportamiento reproductivo de la especie, observándose apareamiento también entre machos macrópteros y hembras braquípteras o viceversa. Se evidenció posturas de huevos alrededor de la nervadura central de las hojas, interior de las vainas foliares, brácteas que envuelven la panoja y brácteas que envuelven las mazorcas tiernas, variando, así, el hábito de oviposición con la fenología de la planta.

Oviposición: La postura es llevada a cabo en las primeras horas de la mañana; así, las hembras se desplazan desde el interior de las vainas foliares hacia el extremo distal de la hoja de maíz. Los huevos son insertados alrededor de la nervadura central de las hojas y en sus cercanías, tanto en el haz como en el envés. Se observó grupos de hasta seis huevos en hileras, los cuales quedan insertados oblicuamente y cubiertos por una sustancia blanco-cerosa secretada por la hembra, que posteriormente se endurece, dejando manchas blancas en la epidermis del tejido vegetal (Fig. 1 y 2), fácilmente visible al ojo humano. Los huevos recién insertados son transparentes, después se evidencian los 
Cuadro 2

Resumen de la duración del ciclo ontogénico de $P$. maidis en condiciones de cautiverio en el valle de Azapa (en días)

\begin{tabular}{|c|c|c|c|c|c|c|c|c|c|}
\hline $\begin{array}{c}\mathbf{N}^{\mathbf{o}} \\
\text { Micro- } \\
\text { jaula }\end{array}$ & $\begin{array}{l}\text { Desarrollo } \\
\text { Embrionario }\end{array}$ & $\begin{array}{c}\text { Ninfa } \\
\text { I }\end{array}$ & $\begin{array}{c}\text { Ninfa } \\
\text { II }\end{array}$ & $\begin{array}{c}\text { Ninfa } \\
\text { III }\end{array}$ & $\begin{array}{l}\text { Ninfa } \\
\text { IV }\end{array}$ & $\begin{array}{c}\text { Ninfa } \\
\mathbf{V}\end{array}$ & Adulto & $\begin{array}{l}\text { Total } \\
\text { Ciclo }\end{array}$ & Sexo \\
\hline 1 & 19 & 10 & 8 & 9 & 11 & 9 & 85 & 80 & oM \\
\hline 2 & 19 & 10 & 11 & 11 & 10 & 11 & 51 & 86 & $\delta^{\top} \mathrm{M}$ \\
\hline 3 & 19 & $*$ & $*$ & $*$ & $*$ & $*$ & $*$ & $*$ & $*$ \\
\hline 4 & 19 & 11 & 7 & 9 & 11 & 12 & 50 & 83 & $\delta^{\top} \mathrm{M}$ \\
\hline 5 & 19 & $*$ & $*$ & $*$ & $*$ & $*$ & $*$ & $*$ & $*$ \\
\hline 6 & 19 & $*$ & $*$ & $*$ & $*$ & $*$ & $*$ & $*$ & $*$ \\
\hline 7 & 23 & $*$ & $*$ & $*$ & $*$ & $*$ & $*$ & $*$ & $*$ \\
\hline 8 & 23 & $*$ & $*$ & $*$ & $*$ & $*$ & $*$ & $*$ & $*$ \\
\hline 9 & 23 & 9 & $*$ & $*$ & $*$ & $*$ & $*$ & $*$ & $*$ \\
\hline 10 & 23 & 9 & $*$ & $*$ & $*$ & $*$ & $*$ & $*$ & $*$ \\
\hline 11 & 23 & 9 & $*$ & $*$ & $*$ & $*$ & $*$ & $*$ & $*$ \\
\hline 12 & 20 & 9 & 9 & 10 & 13 & 15 & 77 & 90 & 우 \\
\hline 13 & 20 & 11 & $*$ & $*$ & $*$ & $*$ & $*$ & $*$ & $*$ \\
\hline 14 & 20 & 10 & 11 & 10 & 12 & 11 & 60 & 88 & $\delta^{\top} \mathrm{M}$ \\
\hline 15 & 23 & $*$ & $*$ & $*$ & $*$ & $*$ & $*$ & $*$ & $*$ \\
\hline 16 & 25 & 12 & 11 & 9 & 11 & 14 & 44 & 96 & $\delta^{\top} \mathrm{M}$ \\
\hline 17 & 19 & 9 & 7 & 11 & 10 & 13 & 57 & 83 & 우 $\mathrm{M}$ \\
\hline 18 & 19 & 11 & 7 & 8 & 9 & 14 & 53 & 82 & $\delta^{\pi} \mathrm{M}$ \\
\hline 19 & 20 & $*$ & $*$ & $*$ & $*$ & $*$ & $*$ & $*$ & $*$ \\
\hline 20 & 25 & 11 & 7 & 9 & 9 & 12 & 86 & 87 & 우 $\mathrm{M}$ \\
\hline 21 & 25 & $*$ & $*$ & $*$ & $*$ & $*$ & $*$ & $*$ & $*$ \\
\hline 22 & 23 & 11 & 14 & 11 & 6 & 10 & 82 & 89 & $\sigma^{\pi} \mathrm{B}$ \\
\hline 23 & 23 & $*$ & $*$ & $*$ & $*$ & $*$ & $*$ & $*$ & $*$ \\
\hline 24 & 25 & 12 & 8 & 10 & 8 & 14 & 66 & 91 & $\delta \mathrm{M}$ \\
\hline 25 & 25 & 11 & 8 & 12 & 12 & 9 & 76 & 91 & $\delta \mathrm{B}$ \\
\hline 26 & 25 & 8 & 9 & 10 & 11 & 8 & 59 & 85 & 우 M \\
\hline 27 & 20 & 10 & 7 & 9 & 11 & 9 & 47 & 80 & 우 $\mathrm{M}$ \\
\hline 28 & 20 & 7 & 8 & 11 & 9 & 10 & 51 & 79 & o $\mathrm{M}$ \\
\hline 29 & 20 & 11 & 9 & 10 & 12 & 9 & 63 & 85 & $\delta^{\pi} \mathrm{B}$ \\
\hline 30 & 23 & 10 & 11 & 10 & 11 & 8 & 48 & 87 & $\delta \mathrm{M}$ \\
\hline $\mathrm{n}$ & 30 & 21 & 17 & 17 & 17 & 17 & 17 & 17 & \\
\hline $\bar{X}$ & 21,63 & 10,05 & 8,94 & 9,88 & 10,35 & 11,06 & 62,1 & 86 & \\
\hline $\begin{array}{c}\text { Desviación } \\
\text { estándar }\end{array}$ & 2,34 & 1,28 & 2,02 & 1,11 & 1,73 & 2,30 & 14,13 & 4,6 & \\
\hline Moda & 23 & 11 & 7 & 10 & 11 & 9 & 51 & 80 & \\
\hline
\end{tabular}

* Ninfas Muertas; + M = Hembra Macróptera; $\delta^{\nearrow} \mathrm{M}=$ Macho Macróptero; $ð^{\top} \mathrm{B}=$ Macho Braquíptero. 
ojos compuestos, pasando de color blanquecino a naranja. El mecanismo para introducir los huevos dentro del tejido de la hoja se facilita mediante el ovipositor en forma de sierra que posee la hembra; es usado para evitar la deshidratación de los mismos. Además, el lugar específico donde ocurrió la oviposición no es al azar, sino que la hembra primeramente hacía contacto con su aparato bucal sobre la epidermis del tejido vegetal; esta operación la realizó cuantas veces fue necesario, cerciorándose, así, que dicho lugar tenía características ideales para el desarrollo del huevo, posteriormente encorva su abdomen en un ángulo de $45^{\circ}$, para finalmente insertar su ovipositor en el lugar escogido. Este comportamiento podría coincidir con lo observado bajo microscopio óptico por Brentassi \& Marino de Remes Lenicov (1999) en muestras de tejido vegetal con presencia de oviposición de Delphacodes kuscheli (Hemiptera: Delphacidae), señalando que los lugares elegidos por este delfácido fueron cavidades aeríferas, y cuando no existían estas cavidades los huevos fueron localizados en tejido parenquimático de plantas de cebada forrajera, Hordeum vulgare L. cv. Bordenave Ranquelina.

En algunas hojas se observó que en las zonas de oviposición de $P$. maidis el tejido se tornó de color morado, fenómeno que algunos autores señalan que se trata de sustancias ovicidas sintetizadas por la planta (Brentassi \& Marino de Remes Lenicov, 1999).

Observaciones sobre la Etología de Peregrinus maidis: La eclosión de los huevos del delfácido fue en las primeras horas de la mañana, con temperaturas bajas, alta humedad relativa y casi nula

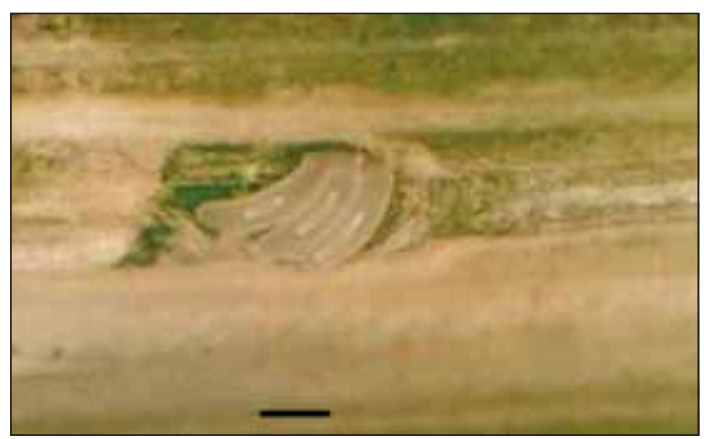

Figura 1. Huevos de P. maidis, insertados por el envés dentro del tejido de la hoja. Vista por el haz. Barra $=0,35 \mathrm{~mm}$.

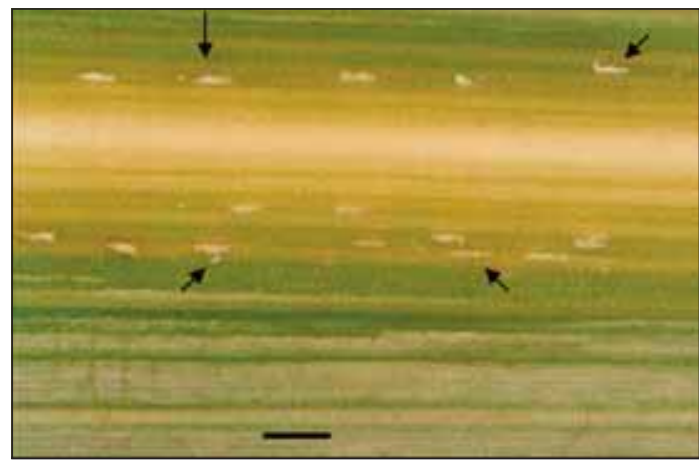

Figura 2. Evidencia de oviposición de P. maidis en las cercanías de la nervadura central de la hoja. Vista por el haz. Barra = $0,4 \mathrm{~mm}$.

presencia de vientos. Al emerger, las ninfas se quedan inmóviles (Fig. 3), luego de recuperarse y tras endurecer su cutícula se desplazan hacia el interior de las vainas foliares; así, se agrupan formando colonias numerosas. También se localizan en el ápice de la planta. Las ninfas son activas y al ser perturbadas se mueven en todas direcciones con movimientos de costado (característico de la especie), también saltan. Para mudar las ninfas fijan las patas en la epidermis de las hojas y luego de 13 a 25 minutos dejan los exuvios.

Los adultos se observan en las hojas, ápice y vainas foliares. Durante la alimentación, excretan abundante líquido azucarado (mielecilla) que cubre la superficie de las hojas, tallos, inflorescencia masculina, brácteas de la mazorca, provocando la aparición de los hongos de la fumagina (Capnodium).

P. maidis necesita unos 132 días para completar su ciclo de vida, requiere alrededor de 71 días para alcanzar su forma adulta. Se debe enfatizar que estos resultados dependieron de la temperatura y humedad relativa entre mayo-septiembre del 2003. Además, estos resultados fueron obtenidos con individuos encerrados en microjaulas, lo que no es completamente equivalente a las condiciones de microclima que presentan el interior de las vainas foliares y ápices de las plantas de maíz, donde desarrolla sus colonias naturalmente.

Enemigos Naturales: En las observaciones periódicas efectuadas en el cultivo de maíz se constató la presencia de predadores generalistas como arácnidos, Nabis sp. (Nabidae); además de larvas de Neurópteros pertenecientes a las familias Chry- 


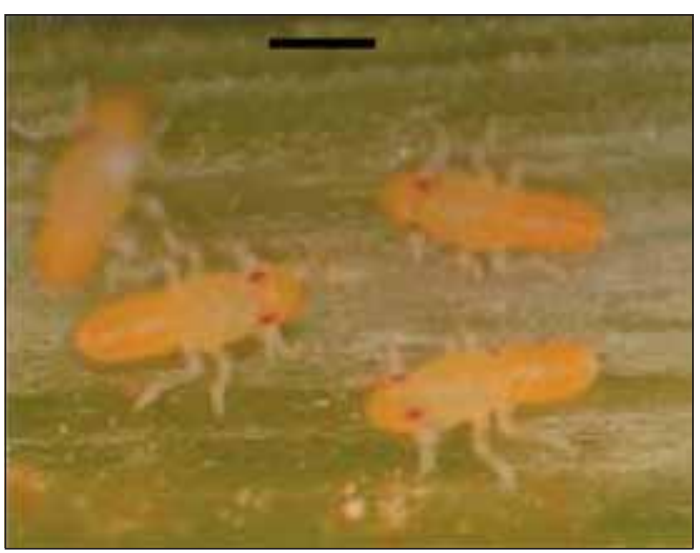

Figura 3. Ninfas de primer instar de P. maidis. Barra $=0,38 \mathrm{~mm}$.

sopidae y Hemerobiidae; de material colectado en el ensayo del 2003 se pudo comprobar la presencia de la avispita Anagrus sp. (Hymenoptera: Mymaridae), parasitoide de huevos de $P$. maidis (Figuras 4 y 5). Además de parasitar sus huevos se alimenta de los melados que excreta el delfácido del maíz. No se registró ningún parásito de ninfas o adultos de $P$. maidis en ambos cultivos.

Plantas Hospedantes: Las malezas presentes en el cultivo y sus alrededores fueron las siguientes: Eragrostis sp. (Poaceae), Setaria verticillata (L.) Beauv (Poaceae), Solanum nigrum L. (Solanaceae), Melilotus indicus (L.) All. (Fabaceae), Medicago sp. (Fabaceae), Euphorbia sp. (Euphorbiaceae), Chenopodium album L. (Chenopodiaceae), Senecio vulgaris L. (Asteraceae), Avena fatua L. (Poaceae), Agropirum repens (L.) Beauv (Poaceae), Sonchus sp. (Asteraceae), Pitraea cuneato - ovata (Cav.) Caro (Verbenaceae), Cynodon dactylon (L.) Pers. (Poaceae), Lolium perenne L. (Poaceae), Sorghum halepense (L.) Pers. (Poaceae).

Se comprobó que, bajo las condiciones agroecológicas locales, $P$. maidis no utiliza plantas hospedantes alternativas sino que desarrolla su ciclo ontogénico exclusivamente en la planta de maíz. En forma esporádica, se colectó ejemplares de este delfácido en Setaria verticillata y Pitraea cuneato-ovata, sin formar colonias en estas especies de plantas. Mientras que en Sorghum halepense -una maleza común en el valle de Azapa- no se detectó presencia de adultos de $P$. maidis, ni indicio alguno de colonias o signos de ataque de este insecto.

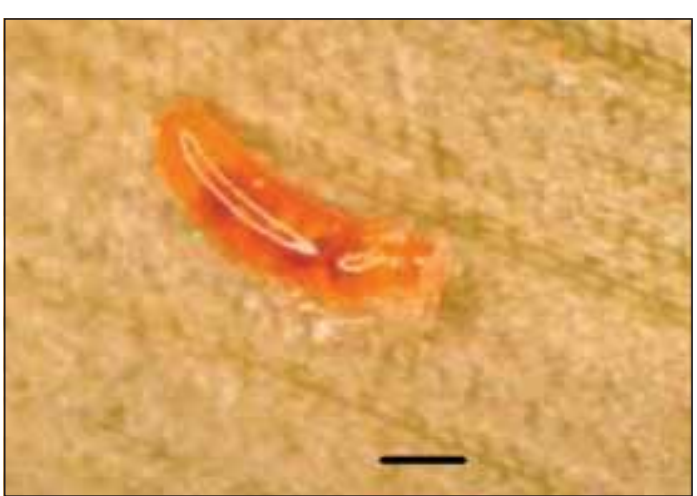

Figura 4. Huevo de P. maidis parasitado por Anagrus sp. Barra $=0,19 \mathrm{~mm}$.

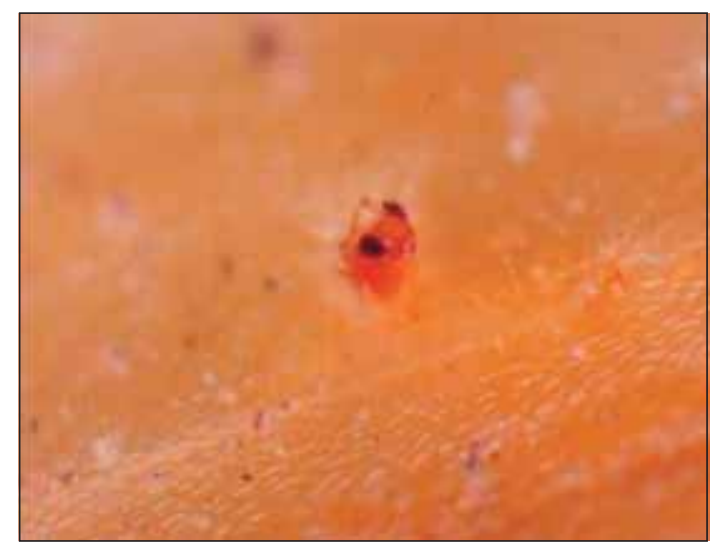

Figura 5. Anagrus sp. emergiendo de un huevo parasitado de P. maidis.

\section{CONCLUSIONES}

Bajo las condiciones climáticas del valle de Azapa, el desarrollo ontogénico de Peregrinus maidis (huevo-adulto) se completa aproximadamente en 70 días; el período de preoviposición dura dos semanas, y el de oviposición se extiende por 15 días. Por lo tanto, el ciclo vital efectivo demora alrededor de 99 días. Los adultos bajo observación presentaron un promedio de 33 días adicionales de vida, contados desde el término de la postura efectiva de huevos. Sin embargo, no se determinó si durante este período las hembras podrían mantener, eventualmente, algún grado de fertilidad adicional, no expresado bajo las condiciones del estudio. Desde un punto de vista teórico, el acrecentamiento de la longevidad posibilita un aumento en el número de oviposturas, y facilita también la dispersión. El ciclo total de vida promedió 132 días (Figura 6). 


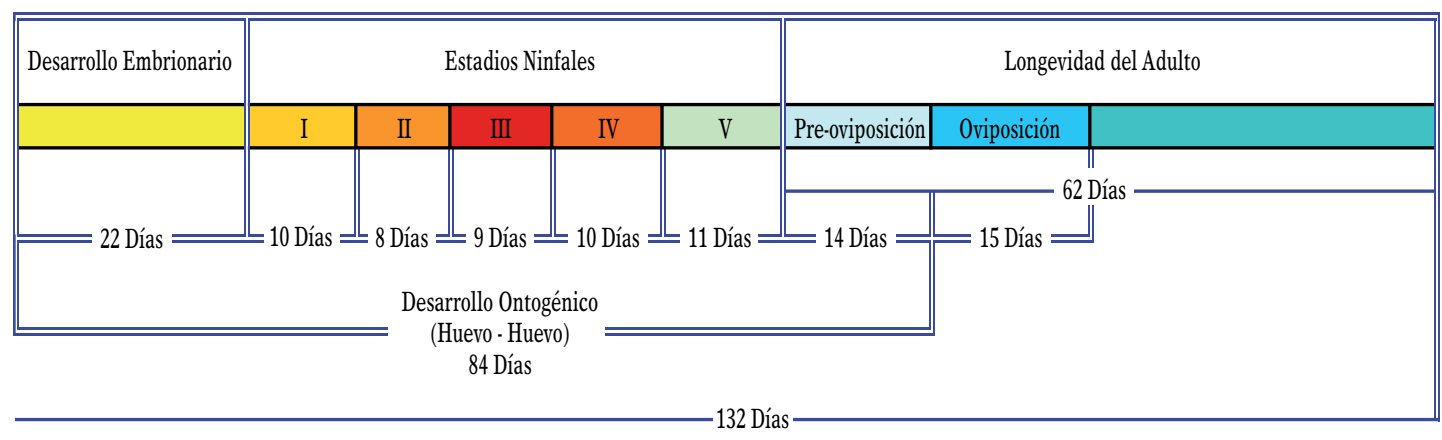

Figura 6. Ciclo Biológico de $P$. maidis en condiciones de cautiverio en el valle de Azapa, km 12.

La ubicación endofítica de los huevos de $P$. maidis, dentro del tejido de la hoja de maíz, asegura un microambiente estable para el desarrollo embrionario, pero no es un escollo para la parasitación por el oófago Anagrus sp. (Hym., Mymaridae), que es el enemigo natural más específico encontrado en el presente estudio.

En el agroecosistema del valle de Azapa, $P$. maidis se comporta como un monófago estricto, desarrollando colonias reproductivas sólo en el cultivo del maíz. Las ninfas neonatas buscan inmediato refugio en el interior de las vainas de las hojas o dentro de la envoltura del ápice del tallo. En los cultivares de Zea mays var amylacea, que son de largo período vegetativo ( $>140$ días) e.g. cv. "Lluteño", P. maidis alcanza a desarrollar dos generaciones. En cambio en los cultivares de maíz dulce, Z. mays var. saccharata, que

\section{LITERATURA CITADA}

AGUILERA, A. 1972. La colección entomológica del Centro de Investigación y Capacitación Agrícola (CICA), Arica. Nuevas determinaciones. II Contribución. IDESIA (Chile) 2: 99-116.

ALATA, J. 1973. Lista de insectos y otros animales dañinos a la agricultura en el Perú. Ministerio de Agricultura del Perú. Dirección general de investigación agraria. Manual No 38 . $176 \mathrm{p}$.

BORROR, D. J.; DELONG, D. M. \& TRIPLEHORN, C. A. 1976. An Introduction to the Study of Insects. $4^{\text {th }}$. Edition. Holt, Rinehart and Winston. U.S.A. 852 p.

BRENTASSI, M. E. \& MARINO DE REMES LENICOV, A. N. 1999. Oviposición de Delphacodes kuscheli (Homoptera-Delphacidae) sobre plantas de cebada en condiciones de laboratorio. Rev. Fac. Agron., La Plata 104 (1): 67-74.

CARVER, M.; GROSS, G. F. \& WOODWARD, T. E. 1991. HEMIPTERA (Bugs, leafhoppers, cicadas, aphis, scale insects etc.). In: The insects of Australia. Vol I. Cornell University Press, Ithaca, New York. 443 pp. son precoces (< 90 días), P. maidis sólo desarrolla una generación.

En el norte de Chile no se cultiva el sorgo, Sorghum vulgare L., pero en el valle de Azapa es común la maleza introducida conocida como maicillo o sorgo de Alepo, Sorghum halepense (L.) Pers. De acuerdo a lo observado, esta planta no es hospedante de $P$. maidis, lo cual podría indicar que las poblaciones locales de P. maidis no han desarrollado los atributos necesarios para romper las barreras de resistencia natural (aleloquímicos) de S. halepense. En cambio, en la relación planta-insecto, se observa una clara interacción trófica, Zea mays - Peregrinus maidis, manteniendo ambas especies una sincronía fenológica, en los sucesivos cultivos de maíz amiláceo, que en los valles costeros de Arica se realizan durante todo el año, desde épocas precolombinas.

CISNEROS, F. 1995. Control de Plagas Agrícolas. $2^{\text {a }}$ Edición. Lima, Perú. 313 p.

CUEZZO, F. \& VIRLA, E. 2001. Interacción entre Delphacidae e Hymenoptera en cultivos de maíz en la Argentina. Revista Sociedad Entomológica Argentina 60 (1-4): 35-37.

FERNÁNDEZ-BADILLO, A \& CLAVIJO, S. 1990. Biología de la Chicharrita del Maíz, Peregrinus maidis (HOMOPTERA: Delphacidae), en Venezuela. Revista de la Facultad de Agronomía (Maracay), 16 (1): 35-46.

FLETCHER, M. \& LARIVIÈRE, M.-C. 2003. Identification Keys and checklists for the leafhoppers, planthoppers and their relatives occurring in Australia and New Zealand (Hemiptera: Auchenorrhyncha). Consulta: 26 de octubre del 2003.<http://www.agric.nsw.gov.au/Hort/ascu/ start.htm>.Última modificación 24 de octubre de 2003.

KING, B. S. \& SAUNDERS, J. L. 1984. Las Plagas Invertebradas de Cultivos Anuales Alimenticios en América Cen- 
tral. Publicado por la administración de desarrollo extranjero (ODA) Londres, UK. 182 p.

MARÍN, R. \& SARMIENTO, J. 1981. Biología y secuencia estacional de Peregrinus maidis. Revista Peruana de Entomología 24 (1): 107-112.

REMES LENICOV, A. M. M. DE \& VIRLA, E. G. 1999. Delfácidos Asociados al Cultivo de Maíz en la República Argentina (Insecta - Homoptera - Delphacidae). Revista de la Facultad de Agronomía, La Plata 104 (1): 1-15.

SCHMUTTERER, H. 1990. Plagas de las Plantas Cultivadas en el Caribe. Con consideración particular en la República
Dominicana. Publicado por la Deutsche Gesellschaft für Technische Zusammenarbeit (GTZ) GMBH, República Federal de Alemania. 640 p.

TSAI, J.H. \& PERRIER, J. L. 1993. Morphology of the digestive and reproductive systems of Peregrinus maidis (Homoptera: Delphacidae). Florida Entomologist 76(3): 428-436.

WILSON, M.R. \& CLARIDGE, M.F. 1991. Handbook for the Identification of Leafhoppers and Planthoppers of Rice. C.A.B. International, Wallingford, UK. 142 p. 